J. Austral. Math. Soc. 20 (Series A) (1975), 172-177.

\title{
SEMIGROUPS IN RINGS
}

\author{
J. CRESP* and R. P. SULLIVAN
}

(Received 16 August 1973)

Communicated by T. E. Hall

\section{Introduction}

A subset $S$ of a ring $R$ is a left semigroup ideal of $R$ if $R S \subseteq R$, and a left ring ideal of $R$ if in addition $S$ is a subring of $R$. Gluskin (1960) investigated those rings with 1 which possess the property:

( $\lambda$ ) every left semigroup ideal is a left ring ideal.

Here we study those rings in which every subsemigroup is a subring, and those in which every semigroup endomorphism is a ring endomorphism. We note in passing that recent work on a rather different, but nonetheless related, question: to characterise certain types of semigroups admitting a ring structure, is to be found in Peinado (1970), Satyanarayana (1971) and Satyanarayana (1973).

\section{Subsemigroups of Rings}

A subset $S$ of $R$ will be called a subsemigroup of $R$ if it is a subsemigroup of $(R, \cdot)$. As usual, for $x \in R,\langle x\rangle$ denotes the cyclic subsemigroup of $R$ generated by $x$. We start by characterising the rings $R$ with the property:

( $\sigma$ ) every subsemigroup of $R$ is a subring.

THeOrem 1. A ring $R$ has $(\sigma)$ iff either $|R|=1$ or $|R|=2$ and $R^{2}=0$.

Proof. Suppose $R$ has $(\sigma)$ and that $|R|>1$. Choose $x \in R \backslash 0$ and consider $\langle x\rangle$. Then from $(\sigma)$, there exists $r \geqq 2$ such that $x^{r}=0$ : suppose $r$ is the least such integer. Now we also have $x+x^{2}=x^{t}$ for some $t>0$. If $r>2$ and $t \geqq 2$, we have $x^{r-2}\left(x+x^{2}\right)=x^{t+r-2}$ and so $x^{r-1}=0$, contradicting the choice of $r$. Hence $r>2$ implies $t=1$ : but then $x^{2}=0$, again contradicting the choice of $r$. Hence $r=2$, and we have $x^{2}=0$ for all $x \in R$.

* The work for this paper was begun while a final-year undergraduate student under the supervision of the second author. 
In particular, for each $y \in R, x y+y x=(x+y)^{2}=0$ and $\{0, x\}$ is a subsemigroup of $R$ for each $x \in R \backslash 0$. Hence from $(\sigma)$, we have $x+x=0$ for all $x \in R$ and so $x y+x y=0$ for all $y \in R$; it follows that $R$ is commutative.

Now suppose $x, y \in R \mid 0$. Since $\{0, x, x y\}$ is a subsemigroup of $R$, and $R$ has $(\sigma)$, we see that $x+x y$ equals $0, x y$, or $x$. In the first case, we obtain $x=x y$ (since $x+x=0$ ) and so $0=x y^{2}=x y$ and $x=0$, a contradiction. If $x+x y$ $=x y$, then $x=0$ trivially and we again obtain a contradiction. So we must have $x+x y=x$, in which case $x y=0$. But then $\{0, x, y\}$ is a subsemigroup of $R$ and so from $(\sigma)$ we deduce that $x+y$ equals $x, y$ or 0 . In the first two cases, either $x$ or $y$ will equal 0 , a contradiction in either case. Hence, $x+y=0$ and so $x=y$; that is, $|R \backslash 0|=1$ and the result follows.

For the converse, suppose $R=\{0, a\}, a \neq 0$, and $a^{2}=0$. Then $a+a$ must equal 0 , and so the subsemigroups $\{0\}$ and $\{0, a\}$ are seen to be subrings.

In the light of the above proof, we now weaken $(\sigma)$, and consider those rings $R$ with:

$\left(\sigma^{\prime}\right)$ every subsemigroup containing 0 is a subring.

In order to characterise all such rings containing 1, we shall need two lemmas: the first summarises Theorems 1 and 3 of Gluskin (1960); the proof of the second can be readily deduced from standard results on finite fields (see Burton (1970)).

LEMMA 1. If $R$ is a ring with 1 which satisfies $(\lambda)$ and $G$ denotes the group of units in $R$, then $R=G \cup G+1$.

LEMma 2. If $F$ is a finite field and a generator of $F \backslash 0$ has order $q$ where $q$ is odd, then $|F|=2^{m}$ for some $m \geqq 1$.

THEOREM 2. A ring $R$ containing 1 has $\left(\sigma^{\prime}\right)$ iff it is a finite field such that $|R| 0 \mid$ is a prime number.

Proof. Since $\left(\sigma^{\prime}\right)$ implies $(\lambda)$, we deduce from Lemma 1 that $R=G \cup G+1$ where $G$ is the group of units in $R$. But $G \cup 0$ is a subsemigroup of $R$, and so from $\left(\sigma^{\prime}\right), G \cup 0$ is a subring. In particular, since $1 \in G$, we have $G+1 \subseteq G \cup 0$ and so $R=G \cup 0$, a division ring.

Now $\{0,1\}$ is a subsemigroup of $R$ and so $\left(\sigma^{\prime}\right)$ implies that $1+1=0$. Hence $x+x=0$ for all $x \in R$. Suppose there exists $x \in R \backslash\{0,1\}$ : we note that if $R=\{0,1\}$, then it is a field of the required type. Then $S=\langle 1+x\rangle \cup\{0,1\}$ is also a subsemigroup of $R$, and so $\left(\sigma^{\prime}\right)$ implies that $x=1+(1+x)=(1+x)^{t}$ for some $t>1$. But $T=\langle x\rangle \cup\{0,1\}$ is another subsemigroup of $R$ and so again using $\left(\sigma^{\prime}\right)$ we obtain $1+x=x^{5}$ for some $s>1$. Hence, for each $x \in R \backslash\{0,1\}$, there exists $r>1$ such that $x^{r}=1$, and so from Jacobson's Theorem (see Burton (1970)) we deduce that $R$ is a field.

If $x \in R \backslash\{0,1\}$, let $q$ be the least integer such that $x^{q}=1$, and suppose 
$q=2 k$. Then $\left\{0,1, x^{k}\right\}$ is a subsemigroup in which, by choice of $q, x^{k} \neq 1$. But by applying $\left(\sigma^{\prime}\right)$ we obtain a contradiction. Hence $q$ is odd, and $T=\langle x\rangle \cup 0$ is a finite field with a generator having odd order. By Lemma $2,|T|=2^{m}$ for some $m \geqq 1$ and $q=2^{m}-1$; we assert that in addition $q$ is prime.

For, suppose $q=a b$ : since $q$ is odd, both $a, b$ are odd. Then $\langle x\rangle$ will contain subgroups $A=\left\langle x^{a}\right\rangle$ and $B=\left\langle x^{b}\right\rangle$ of order $b$ and $a$ respectively, and so by $\left(\sigma^{\prime}\right), A \cup 0$ and $B \cup 0$ are finite fields, each with a generator having odd order. Therefore by Lemma 2 , there exists $u, v \geqq 1$ such that $a=2^{u}-1, b=2^{v}-1$. Hence

$$
2^{m}-1=q=a b=\left(2^{u}-1\right)\left(2^{v}-1\right)
$$

and so

$$
2^{m-1}-1=2^{u+v-1}-2^{u-1}-2^{v-1}
$$

a contradiction if both $u, v>1$. So either $u \leqq 1$ or $v \leqq 1$, and hence either $a=1$ or $b=1$.

We have now shown that for each $x \in R \backslash\{0,1\}, x$ has odd prime order. Suppose $x \in R \backslash\{0,1\}$ and there exists a non-zero $y \in R \mid\langle x\rangle$. Then $x y \in R \backslash\{0,1\}$ and if $x, y$ have prime order $p, q$ respectively, then $x y$ has non-prime order $p q$, a contradiction. Hence $R=\langle x\rangle \cup 0$ is a finite field of order $2^{m}$ for which $2^{m}-1$ is prime.

Suppose conversely that $R$ is such a field. Then $\langle x\rangle$ is a cyclic group of prime order, and so any element of $\langle x\rangle$ is a generator of $\langle x\rangle$. Hence if $S$ is a subsemigroup of $R$ containing 0 and if there exists $x \in S \backslash\{0,1\}$, then $S=R$ is certainly a subring; that is, $R$ has $\left(\sigma^{\prime}\right)$, and the proof is complete.

\section{Semigroup endomorphisms}

A semigroup endomorphism of a $\operatorname{ring} R$ is a mapping $\phi: R \rightarrow R$ such that $(x y) \phi=x \phi \cdot y \phi$ for all $x, y \in R$; a ring endomorphism is a semigroup endomorphism $\phi: R \rightarrow R$ such that $(x+y) \phi=x \phi+y \phi$ for all $x, y \in R$. We start by considering the following property of a ring $R$.

$(\varepsilon)$ every semigroup endomorphism is a ring endomorphism.

THEOREM 3. If $R$ is a commutative ring with (c), then either (i) $|R|=1$, or (ii) $|R|=2$ and $R^{2}=0$, or (iii) $R=R^{2}$ and $a+a=0=a^{2}$ for all $a \in R$.

Proof. Consider the mapping $\theta_{n}: R \rightarrow R$ defined by setting $x \theta_{n}=x^{n}$ for all $x \in R$. Since $R$ is commutative, $\theta_{n}$ is a semigroup endomorphism for each $n \geqq 1$, and hence since $R$ has $(\varepsilon)$, each $\theta_{n}$ is a ring endomorphism. Then putting $n=2$, we obtain $x y+x y=0$ for all $x, y \in R$, and from $n=3$, we obtain $x y^{2}+x^{2} y=0$. But then $x^{2}+x^{2}=0$ and so $x y^{2}=x^{2} y$ for all $x, y \in R$. In particular, $x^{5}=x^{4}$ when $y=x^{2}$, and so $x^{4}$ is an idempotent. Now fix $a \in R$ and define $y: R \rightarrow R$ 
by setting $x \gamma=a^{4}$ for all $x \in R$. Since $\gamma$ is clearly a semigroup endomorphism, we deduce from $(\varepsilon)$ that $a^{4}=0$ for all $a \in R$.

Now suppose $a \in R \mid 0$ and define $\tau_{a}: R \rightarrow R$ and $\mu_{a}: R \rightarrow R$ by

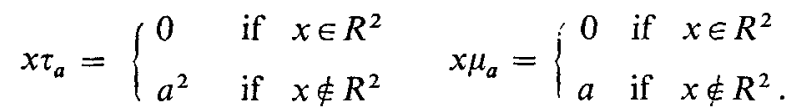

If $x, y \in R$, then $x y \in R^{2}$ and so $(x y) \tau_{a}=0$. If either $x \in R^{2}$ or $y \in R^{2}$, then $x \tau_{a} \cdot y \tau_{a}=0$, and if both $x, y \notin R^{2}$, then $x \tau_{a} \cdot y \tau_{a}=a^{2} \cdot a^{2}=0$. Hence $\tau_{a}$ is a semigroup endomorphism, and so by $(\varepsilon)$, is a ring endomorphism. Suppose $v \in R^{2}$ and $u \notin R^{2}$. If $v+u \in R^{2}$, then

$$
a^{2}=v \tau_{a}+\mu \tau_{a}=(v+u) \tau_{a}=0 .
$$

We assert that now $\mu_{a}$ is a semigroup endomorphism. For if $x, y \in R$, then $x y \in R^{2}$ and $(x y) \mu_{a}=0$, and if either $x \in R^{2}$ or $y \in R^{2}$, then $x \mu_{a} \cdot y \mu_{a}=0$; if $x, y \notin R^{2}$, then $x \mu_{a} \cdot y \mu_{a}=a \cdot a=0$. From $(\varepsilon)$ we now deduce that

$$
a=v \mu_{a}+u \mu_{a}=(v+u) \mu_{a}=0,
$$

a contradiction. Hence if $u \notin R^{2}$ and $v \in R^{2}$, then $u+v \notin R^{2}$.

Now suppose there exists $c \in R \backslash R^{2}$ and define $\kappa_{a}: R \rightarrow R$ and $\lambda_{a}: R \rightarrow R$ by

$$
x \kappa_{a}=\left\{\begin{array}{ll}
0 & \text { if } x \in R^{2} \cup c \\
a^{2} & \text { otherwise }
\end{array} \quad x \lambda_{a}= \begin{cases}0 & \text { if } x \in R^{2} \cup c \\
a & \text { otherwise. }\end{cases}\right.
$$

If $x, y \in R$, then $x y \in R^{2} \cup c$, and so $(x y) \kappa_{a}=0$. If either $x \in R^{2} \cup c$ or $y \in R^{2} \cup c$, then $x \kappa_{a} \cdot y \kappa_{a}=0$, and if both $x, y \notin R^{2} \cup c$, then $x \kappa_{a} \cdot y \kappa_{a}=a^{2} \cdot a^{2}=0$. Hence $\kappa_{a}$ is a semigroup endomorphism which from $(\varepsilon)$ is also a ring endomorphism. Suppose $u, v \in R^{2} \cup c$ and $u+v \notin R^{2} \cup c$. Then

$$
a^{2}=(u+v) \kappa_{a}=u \kappa_{a}+v \kappa_{a}=0,
$$

and so as in the case of $\mu_{a}$ above, we can deduce that $\lambda_{a}$ is a semigroup endomorphism. But then $(\varepsilon)$ implies that $a=(u+v) \lambda_{a}=u \lambda_{a}+v \lambda_{a}=0$, a contradiction. Hence, if $u, v \in R^{2} \cup c$, then $u+v \in R^{2} \cup c$. In particular, $x y+c \in R^{2} \cup c$ for all $x, y \in R$. But we know from the above that $x y+c \notin R^{2}$. Hence, $x y+c=c$ and so $R^{2}=0$.

Now define $\delta: R \rightarrow R$ by setting $0 \delta=0$ and $x \delta=a$ for all $x \in R \backslash 0$. If $x, y \in R$, we know that $x y=0$ and so $(x y) \delta=0$. If either $x=0$ or $y=0$, then $x \delta \cdot y \delta=0$, and if $x \neq 0$ and $y \neq 0$, then $x \delta \cdot y \delta=a^{2}=0$. Hence $\delta$ is a semigroup endomorphism which is by $(\varepsilon)$ a ring endomorphism. So, if there exist $x, y \neq 0$ in $R$ such that $x-y \neq 0$, then $0=a-a=x \delta-y \delta=(x-y) \delta=a$, a contradiction. Hence, $R=\{0, a\}$ and so (ii) holds. 
Suppose now that $R \backslash R^{2}=\square$; that is, $R=R^{2}$. Then for each $a \in R, a=x y$ for some $x, y \in R$ and so from an earlier comment, $a+a=0$. In addition, $a^{2}=(x y)^{2}=x^{2} y^{2}=\left(x^{2}\right)^{2} y=0$, and so (iii) holds.

REMARK. If $|R|=1$ or if $|R|=2$ and $R^{2}=0$, then $R$ has ( 8 ): it is not known whether rings satisfying condition (iii) in the above theorem exist, nor whether, if they do, they have $(\varepsilon)$.

As in Section 2, we now weaken $(\varepsilon)$, and investigate those rings $R$ with:

$\left(\varepsilon^{\prime}\right)$ every non-constant semigroup endomorphism is a ring endomorphism.

THEOREM 4. If $R$ is a commutative ring with 1 and satisfies $\left(\varepsilon^{\prime}\right)$, then $R$ is a field of order 2.

Proof. Let $\theta_{n}: R \rightarrow R$ be defined by setting $x \theta_{n}=x^{n}$ for all $x \in R$. Then since $R$ is commutative, $\theta_{n}$ is a semigroup endomorphism of $R$, and is non-constant since $1 \theta_{n}=1$ and $0 \theta_{n}=0$ and $0 \neq 1$. Hence $\left(\varepsilon^{\prime}\right)$ implies that $\theta_{2}$ is a ring endomorphism, and so for all $x \in R,(x+1)^{2}=(x+1) \theta_{2}=x^{2}+1$; that is, $x+x=0$ for all $x \in R$. Likewise $\theta_{3}$ is a ring endomorphism, and for all $x \in R$

$$
(x+1)^{3}=(x+1) \theta_{3}=x^{3}+1 ;
$$

that is, $x^{2}+x=0$ (since $x+x=0$ ) and so $x=x^{2}$ for all $x \in R$. Hence $R$ is Boolean.

Now let $a \in R \backslash\{0,1\}$ and define $\gamma: R \rightarrow R$ by:

$$
\begin{aligned}
& x y=a \text { if } x a \neq a \\
& =x \text { if } x a=a
\end{aligned}
$$

We assert that $\gamma$ is a non-constant semigroup endomorphism of $R$. For, suppose $x, y \in R$ and $x y a \neq a$. Then $(x y) \gamma=a$ and we may suppose without loss of generality that $x a \neq a$ : if $x a=a=y a$, then $x y a=a$ since $R$ is Boolean and this is a contradiction. So, $x \gamma \cdot y \gamma=a \cdot y \gamma$ and this equals $a \cdot a$ if $y a \neq a$ or $a y$ if $y a=a$ : therefore in either case $x y \cdot y \gamma=a=(x y) y$. If on the other hand $x y a=a$, then $x a=x^{2} y a=x y a=a$ and $y a=a$ similarly. Hence in this case also, $(x y) \gamma=x y=x \gamma \cdot y \gamma$. Finally, $\gamma$ is non-constant since for example $1 \gamma=1$ and $0 \gamma=a \neq 1$, and so our assertion holds. But now $\left(\varepsilon^{\prime}\right)$ implies that $\gamma$ is a ring endomorphism and so in particular $0 \gamma=0$; that is, $a=0$, a contradiction. Hence there is no such $a$ in $R$ and we have $|R|=2$.

In the above theorem, commutativity of $R$ was essentially used to establish the existence of certain semigroup endomorphisms defined on $R$ : it is unknown whether an arbitrary ring, with or without 1 , has at least one semigroup endomorphism that can be defined on $R$ in some algebraic manner. The next result replaces the criterion of commutativity by one suggested by Mr. J. S. V. Symons: it holds in, for example, all full matrix rings. 
THEOREM 5. If $R$ is a ring with 1 in which every 1-sided unit is 2-sided and which has $\left(\varepsilon^{\prime}\right)$, then $R$ is a field of order 2 .

Proof.Let $L$ be the set of left-units, and $G$ the set of units, in $R$. Define $\gamma: R \rightarrow R$ by:

$$
x y=\left\{\begin{array}{lll}
x & \text { if } & x \in G \\
0 & \text { if } & x \notin G .
\end{array}\right.
$$

We assert that $y$ is a non-constant semigroup endomorphism of $R$. For, if $x, y \in R$ and $x y \in G$, then $(x y) \gamma=x y$ and $x \in L$. Hence $x \in G$ and so $y=x^{-1} x y \in G$. From the definition of $\gamma$, we therefore have

$$
x \gamma \cdot y \gamma=x y=(x y) \gamma .
$$

On the other hand, if $x y \notin G$, then without loss of generality we may assume $x \notin G$. Then

$$
(x y) \gamma=0=0 \cdot y \gamma=x \gamma \cdot y \gamma,
$$

and clearly $1 \gamma=1,0 \gamma=0$, and $1 \neq 0$ imply that $\gamma$ is non-constant. By $\left(\varepsilon^{\prime}\right)$, $\gamma$ is therefore a ring endomorphism. Now suppose $x \in G, y \notin G$. If $x+y \notin G$, we have $x+0=x \gamma+y \gamma=(x+y) \gamma==0$ and $0 \in G$, which is impossible. Hence $x+y \in G$. But then $x+0=x y+y \gamma=(x+y) \gamma=x+y$ and so $R \backslash G=\{0\}$; that is, $R$ is a division ring.

Now define $\lambda: R \rightarrow R$ by setting $0 \lambda=0$ and $x \lambda=1$ for all $x \in R \mid 0$. If $x, y \in R$ and $x y=0$, then $x=0$ or $y=0$, and so $(x y) \gamma=0=x \gamma \cdot y \gamma$. If $x y \neq 0$, then both $x, y \neq 0$ and we have $(x y) \gamma=1=x \gamma \cdot y \gamma$. Hence $\gamma$ is a semigroup endomorphism which is obviously non-constant. By $\left(\varepsilon^{\prime}\right), \gamma$ is therefore a ring endomorphism. Now suppose there exists $x \in R \backslash\{0,1\}$. If $x+x \neq 0$, we have $1+1=x y+x y=(x+x) y=1$, which is impossible. Hence $x+x=0$ and so $1+x \neq 0$. But now $1+1=1 \gamma+x \gamma=(1+x) \gamma=1$. Hence $R=\{0,1\}$ and the result follows.

\section{References}

D. M. Burton (1970), A first course in the theory of rings and ideals (Addison-Wesley, London, 1970).

L. M. Gluskin (1960), 'Ideals in rings and their multiplicative semigroups', Uspedni Mat. Nauk. (N. S.) 15, No. 4 (94), 141-148; translated in Amer. Math. Soc. Translations, 27 (2) (1963), 297-304.

R. E. Peinado (1970), 'On semigroups admitting ring structure', Semigroup Forum 1, 189-208.

M. Satyanarayana (1971), 'On semigroups admitting ring structure', Semigroup Forum 3, 43-50,

M. Satyanarayana (1973), 'On semigroups admitting ring structure II', Semigroup Forum 6, 189-197.

University of Western Australia

Nedlands

W.A. 6009

Australia. 